\title{
Discrete Differential Geometry Applied to the Coil-End Design of Superconducting Magnets
}

\author{
Bernhard Auchmann, Stephan Russenschuck, and Nikolai Schwerg
}

\begin{abstract}
CCoil-end design for superconducting accelerator magnets, based on the continuous strip theory of differential geometry, has been introduced by Cook in 1991. A similar method has later been coupled to numerical field calculation and used in an integrated design process for LHC magnets within the CERN field computation program ROXIE. In this paper we present a discrete analog on to the continuous theory of strips. Its inherent simplicity enhances the computational performance, while reproducing the accuracy of the continuous model. The method has been applied to the design of coil ends for the SIS300 dipole magnets of the FAIR project.
\end{abstract}

Index Terms-Coil winding, differential geometry.

\section{INTRODUCTION}

$\mathbf{T}$ HE SIS300 dipole magnets of the FAIR project will be wound from Rutherford-type cables with a stainless-steel core [1]. The core makes the cable less stable and thus more difficult to wind into a saddle-shaped coil. Winding tests have revealed a very low limit for so-called hard-way bend (bending of the cable over its narrow edge) to avoid collapsing cable and lift-off from the winding mandrel.

In the elastic regime, the strain energy $E$ in a rectangular rod is proportional to the square of the curvature parameters, i.e., torsion $\omega^{1}(s)$, normal curvature $\omega^{2}(s)$, and geodesic curvature $\omega^{3}(s)$ :

$$
E=\frac{1}{2} \int_{0}^{s_{c}}\left(\nu_{1}\left(\omega^{1}\right)^{2}+\nu_{2}\left(\omega^{2}\right)^{2}+\nu_{3}\left(\omega^{3}\right)^{2}\right) \mathrm{d} s,
$$

where $s$ is the arc-length and $s_{\mathrm{c}}$ is the overall cable length. The flexural rigidities of the cable, $\nu_{1}, \nu_{2}, \nu_{3}$, are material and cable-type related parameters to be measured. In general, $\nu_{3} \gg$ $\nu_{1} \nu_{2}$, so that the control of the strain energy can be reduced to controlling the geodesic curvature, with the ideal cable having zero geodesic curvature.

Models for the geometry of cables in coil ends of so-called $\cos \Theta$ magnets have employed the theory of strips, which is a discipline of differential geometry [2], [3]. These models analytically determine the shape of an ideal strip from a parameterized baseline, e.g, an ellipse on the winding mandrel. In this paper we introduce a solely discrete theory of strips. The discrete theory is

Manuscript received August 29, 2006

The authors are with CERN AT/MEL, CH-1211 Geneva 23, Switzerland (e-mail: bernhard.auchmann@cern.ch).

Color versions of one or more of the figures in this paper are available online at http://ieeexplore.ieee.org.

Digital Object Identifier 10.1109/TASC.2007.897233

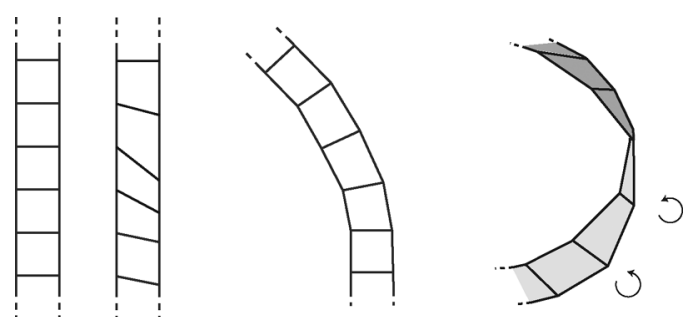

Fig. 1. Left: Two flat and straight discrete strips, differing only by the position of the joints. Right: Bending of a curved strip in its joints.

computationally more efficient and yields a more flexible framework for the modeling of coils and busbars, as no parameterization of the baseline is required. The asymptotical equivalence of discrete and continuous model is demonstrated and the coil-end design for the SIS300 magnet is briefly outlined.

\section{MATHEMATICAL FRAMEWORK}

\section{A. Discrete Strips, Bending, Geodesic Curvature}

We define a discrete strip as a set of flat, quadrilateral faces embedded in $\mathbb{R}^{3}$. Each face has exactly two neighboring faces and is bounded by four edges: we distinguish between the two boundary edges (one of which will become the baseline) and the two joining edges (in short joints) to the neighboring faces.

Bending of a strip is the act of isometric (arc-length preserving) continuous deformation. In the discrete model, bending consists in turning the strip's faces around the joints, a process that is the very definition of a developable surface. Developable surfaces in Euclidean space are surfaces which can essentially be made of a piece of paper, if we assume sufficient smoothness and thus exclude possible ways of arranging crumpled paper.

Fig. 1 (left) shows two straight discretized strips. Fig. 1 (right) illustrates the bending of a non-geodesic strip, that is, a strip with hard-way bend. Obviously, the joints of a discrete strip must not intersect.

The curvature of a flat strip is measured by the angles $\alpha_{i}=$ $\angle\left(\mathbf{d}^{i}, \mathbf{a}_{1}^{i}\right)$ and $\beta_{i}=\angle\left(\mathbf{d}^{i}, \mathbf{a}_{1}^{i+1}\right)$ between the joints represented by the joint vectors $\mathbf{d}^{i}$ and the tangent vectors $\mathbf{a}_{1}^{i}$ of neighboring baseline edges, see Fig. 2 (left). For $\alpha_{i}+\beta_{i}=180^{\circ}$ we call the strip a straight or geodesic strip. The measure

$$
\omega_{i}^{3}=180^{\circ}-\left(\alpha_{i}+\beta_{i}\right)
$$

is called the discrete geodesic curvature or the discrete hard-way bend. The geodesic curvature $\omega^{3}$ is invariant with respect to bending. 


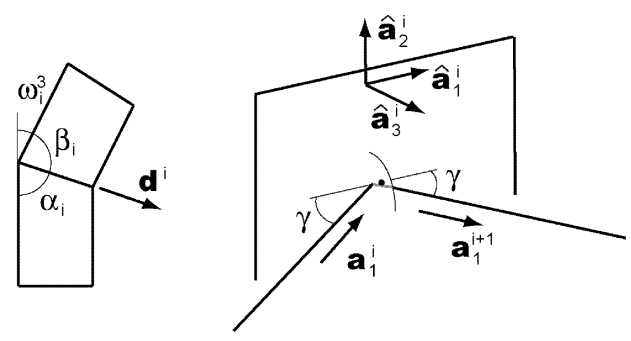

Fig. 2. Left: Illustration of the discrete geodesic curvature measure. Right: Tangent plane assigned to a node of the primary baseline.

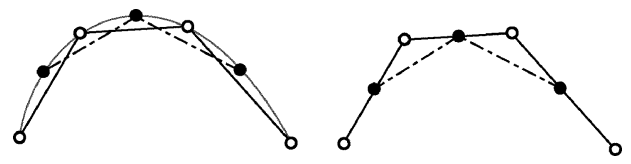

Fig. 3. Primary and secondary (dashed) discrete baseline. For a given parameterization of the baseline, primary and secondary nodes may be located on the baseline (left). If no parametrization of the baseline is given (the input is a mere set of primary nodes), secondary nodes are located in the barycenters of primary edges (right).

Furthermore we define the discretization density $h:=$ $\sup _{i}\left(l_{\mathrm{a}}^{i}\right)$, where $l_{\mathrm{a}}^{i}$ is the length of the baseline element $i$, and $h$ is the length of the longest element in the set.

\section{B. Geodesic Strip}

The aim is now to find an element from the set of geodesic strips that can be bent along a given discrete baseline, i.e., we determine the joints around which the strip can be developed. To this end we introduce two discrete baselines denoted primaryand secondary baseline, see Fig. 3. Let $\mathbf{a}_{1}^{i}$ denote the $i$-th normalized tangent vector to the primary baseline and $\overline{\mathbf{a}}_{1}^{i}$ the normalized tangent vector to the secondary baseline. The primary baseline is used to determine normal vectors to the faces of the strip that is bent along the secondary baseline.

We define the mean tangent vector as

$$
\hat{\mathbf{a}}_{1}^{i}:=\frac{\mathbf{a}_{1}^{i+1}+\mathbf{a}_{1}^{i}}{\left\|\mathbf{a}_{1}^{i+1}+\mathbf{a}_{1}^{i}\right\|},
$$

and the mean normal vector as

$$
\hat{\mathbf{a}}_{3}^{i}:=\frac{\mathbf{a}_{1}^{i+1}-\mathbf{a}_{1}^{i}}{\left\|\mathbf{a}_{1}^{i+1}-\mathbf{a}_{1}^{i}\right\|} .
$$

The (mean) binormal vector is then $\hat{\mathbf{a}}_{2}^{i}=\hat{\mathbf{a}}_{3}^{i} \times \hat{\mathbf{a}}_{1}^{i}$.

Making use of $\overline{\mathbf{a}}_{1}^{i} \approx \hat{\mathbf{a}}_{1}^{i}$, we now use the normal vectors $\hat{\mathbf{a}}_{3}^{i}$ as normal vectors to the secondary faces: $\overline{\mathbf{a}}_{3}^{i}:=\hat{\mathbf{a}}_{3}^{i}$, see Fig. $4 .{ }^{1}$

The joints of the secondary strip are defined as the intersections of two consecutive tangent planes. The joint vector $\overline{\mathbf{d}}^{i}$ in node $i$ of the secondary baseline must therefore be orthogonal to $\overline{\mathbf{a}}_{3}^{i}$ and $\overline{\mathbf{a}}_{3}^{i+1}$. Thus

$$
\overline{\mathbf{d}}^{i}=\overline{\mathbf{a}}_{3}^{i} \times \overline{\mathbf{a}}_{3}^{i+1} .
$$

${ }^{1}$ To justify this choice we show that $\omega_{i}^{\prime}:=\left(\mathbf{a}_{1}^{i+1}-\mathbf{a}_{1}^{i}\right) \cdot \hat{\mathbf{a}}_{2}^{i}=0$ and that $\lim _{h \rightarrow 0} \omega_{i}^{\prime}=\lim _{h \rightarrow 0} \omega_{i}^{3}$. We see that vanishing $\omega_{i}^{\prime}$ implies vanishing geodesic curvature for $h \rightarrow 0$.

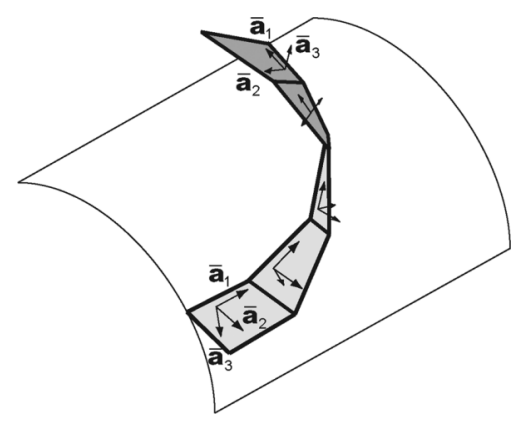

Fig. 4. Moving frame along the faces of a bent strip.

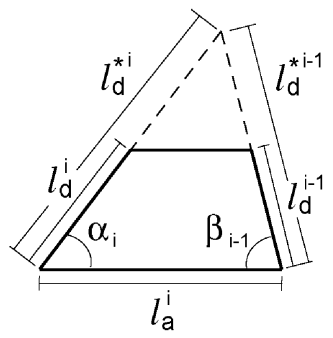

Fig. 5. Calculating the intersection of consecutive joints to verify that this intersection is indeed outside the strip surface.

An example of the resulting bent strip is shown in Fig. 4. For $h \rightarrow 0$ the joint vector is identical to the Darboux vector in differential geometry, [4].

\section{Edge of Regression}

In order to determine the length $l_{\mathrm{d}}^{i}$ of the joints we calculate

$$
l_{\mathrm{d}}^{i}=\frac{1}{2}\left(\frac{w}{\sin \alpha_{i}}+\frac{w}{\sin \beta_{i}}\right) \text {, }
$$

where $\alpha_{i}$ and $\beta_{i}$ are defined in Fig. 2 (left) and where $w$ denotes the width of the strip. In general, only a straight strip will have parallel bounding edges. The family of intersections of consecutive joints is called the edge of regression.

We have to verify whether the resulting joint vectors represent an admissible choice for a strip of a given width $w$. The intersection point of any two consecutive joints, as well as the joint lengths $l_{\mathrm{d}}^{* i}$ and $l_{\mathrm{d}}^{* i-1}$ can be calculated from geometrical data in the plane

$$
\begin{aligned}
l_{\mathrm{d}}^{* i} \cos \alpha_{i}+l_{\mathrm{d}}^{* i-1} \cos \beta_{i-1} & =l_{\mathrm{a}}^{i}, \\
l_{\mathrm{d}}^{* i} \sin \alpha_{i} & =l_{\mathrm{d}}^{* i-1} \sin \beta_{i-1},
\end{aligned}
$$

where $l_{\mathrm{a}}^{i}$ is the length of the baseline element. An edge of regression penetrating into the strip surface indicates a non-physical solution (crumbled and torn paper, so to say). It is thus verified that indeed $l_{\mathrm{d}}^{* i}>l_{\mathrm{d}}^{i}$ and $l_{\mathrm{d}}^{* i-1}>l_{\mathrm{d}}^{i-1}$, compare Fig. 5 .

A violation of the above criteria indicates that no geodesic strip of width $w$ can be bent onto the given baseline. Assuming that the baseline is fixed, some geodesic curvature has to be admitted. In order to avoid crossing joints within the strip, additional twist of the faces around the baseline elements can be 

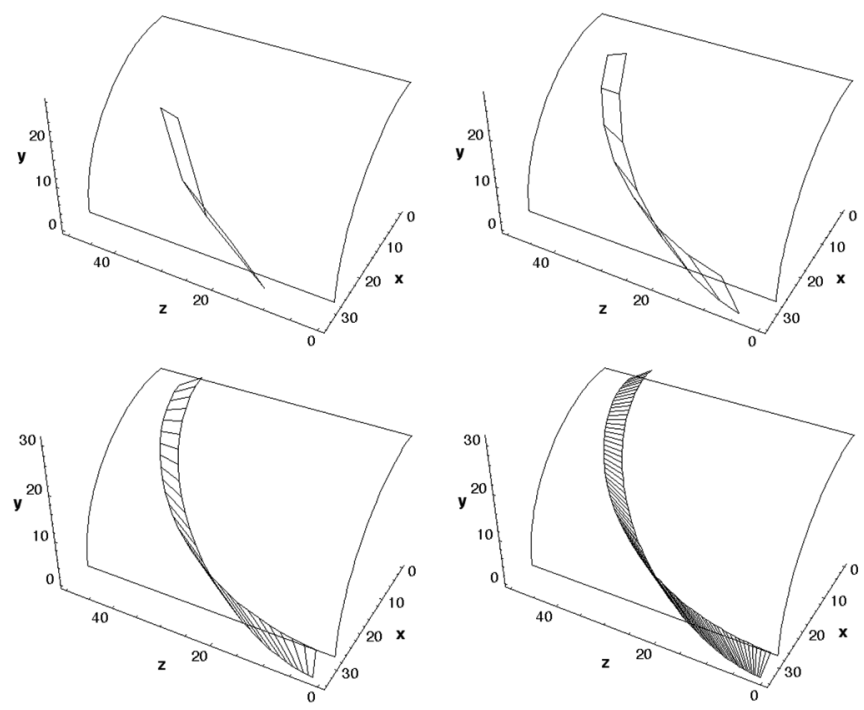

Fig. 6. Discrete strips bent along a discrete "ellipse on a cylinder." The models contain $5,10,30$, and 80 points on the primary baseline. Note that for $n$ nodes on the primary baseline, only $n-2$ joint vectors are mathematically defined in the nodes of the secondary baseline.

applied (and is sometimes required by the non-radial position of the cable at the magnet's cross-section). The additional twist angle is denoted $\tau_{i}$. We obtain

$$
\begin{aligned}
& \overline{\mathbf{a}}_{3}^{\prime i}=\cos \tau_{i} \overline{\mathbf{a}}_{3}^{i}+\sin \tau_{i} \overline{\mathbf{a}}_{2}^{i}, \\
& \overline{\mathbf{a}}_{2}^{\prime i}=\cos \tau_{i} \overline{\mathbf{a}}_{2}^{i}-\sin \tau_{i} \overline{\mathbf{a}}_{3}^{i} .
\end{aligned}
$$

From the primed normal vectors we can calculate the updated joint vectors $\overline{\mathbf{d}}^{i}$ from (5), check the geodesic curvature (2) and the intersection of joints $(7 b)$.

\section{VERIFICATION OF THE MODEL}

In order to verify the discrete model, we determine a discrete geodesic strip that is bent along an "ellipse on a cylinder". The model is shown in Fig. 6 for different discretization densities. Note that the first and the last joint vector to complete the semi-arc are missing, as for $n$ nodes on the baseline, only $n-2$ joint vectors are mathematically defined. The problem can be overcome by making use of the symmetry at the apex of the cylinder and by assuming that the strip continues as a flat, straight strip at the onset of the arc.

The discrete geodesic curvature $\omega^{3}$ as defined in (2) tends to zero with $o\left(h^{3}\right)$ for $h \rightarrow 0$, which is shown for the numerical example in Fig. 7(a).

Comparing the discrete model to a model based on continuous differential geometry, [3] we see that the joint vectors in the discrete model converge to the Darboux-vectors in a continuous model. Fig. 7(b) shows the distribution of the angle $\delta$ between the joint vectors and the Darboux-vectors, which tends to zero with $o\left(h^{2}\right)$ for $h \rightarrow 0$, thus validating the discrete theory.

\section{COIL-END DESIGN IN ROXIE}

The above model has been implemented in the CERN field computation program ROXIE and is linked to an optimization toolkit, [5]. The integrated design process of coil ends now includes the following steps: (a)

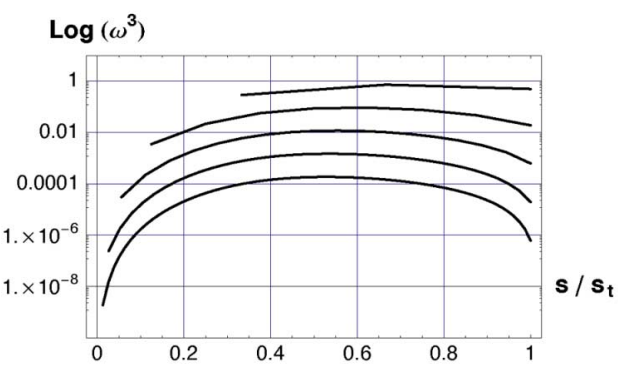

(b)

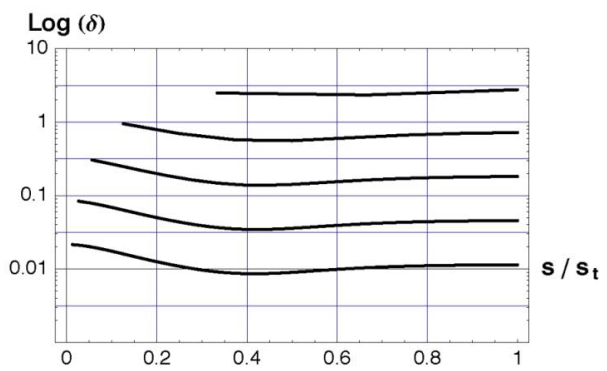

Fig. 7. Logarithmic plot of (a) the geodesic curvature in the discrete strip and (b) of the angle between the discrete joint vectors and the continuous Darboux vectors. The discrete models contain (top-to-bottom graph) 5, 10, 20, 40, and 80 nodes on the primary baseline. The angles are given in degrees.

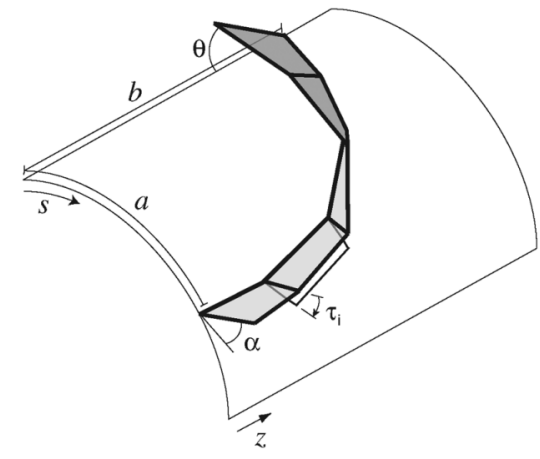

Fig. 8. Display of design variables. $\theta$ is the inclination angle at the apex; $\alpha$ is the inclination angle at the onset of the coil end; $\tau_{i}$ is the additional twist angle by which each face of the discrete strip can be twisted; $b / a$ is the ellipticity of the hyper-ellipse on the winding mandrel.

1) The 2-D cross-section, that has been optimized with the electromagnetic codes of ROXIE, defines the starting point of each cable's baseline on the winding mandrel. We use the hyper-ellipse formula

$$
\frac{s^{k}}{a^{k}}+\frac{z^{2}}{b^{2}}=1
$$

to calculate the points on the primary and secondary baselines. $z$ is the longitudinal coordinate in the magnet and $s$ is the azimuthal arc-length on the winding mandrel as measured from the apex. The ellipticity ratio $b / a$ and the order of the hyper-ellipse $k$ are optimization parameters for the definition of the baselines.

2) We determine the position of the discrete geodesic strip on the baseline of the first cable in a block according to the above theory.

3) The inclination angle $\alpha$ at the onset of the coil end is defined by the 2-D cross-section, compare Fig. 8. The inclination angle $\theta$ at the apex of the mandrel is provided as a 
design variable in order to be able to match two unsymmetric arcs at the apex. The geodesic strip calculated in the previous step generally matches neither of the inclination angles $\alpha$ and $\theta$. An additional twist by an angle $\tau_{i}$ is therefore applied to each face of the strip according to $(8 b)$, which is distributed linearly along the strip is required to match $\alpha$ and $\theta$. This inevitably introduces some geodesic curvature to the strip.

4) A model of all cables wound on top of each other in a coil block is created.

5) Objective function values can be calculated. The integrated square of the geodesic curvature

$$
\left(\omega^{3}\right)^{2}=\sum_{i} \sum_{j}\left(\omega_{i j}^{3}\right)^{2}
$$

where $j$ is the cable number in a block and $i$ is the nodenumber on the baseline, is to be minimized. Intersecting joints within a strip are taken as a constraint in the optimization process. To do so, we introduce the intersection parameter $e$ : For each intersecting joint according to (7b), we add the angle between the normal vectors of the neighboring faces.

$e=\sum_{i} \delta_{i} \arcsin \left(\overline{\mathbf{a}}_{3}^{i} \times \overline{\mathbf{a}}_{3}^{i+1}\right)=\sum_{i} \delta_{i} \arcsin \left\|\overline{\mathbf{d}}^{i}\right\|$,

where $\delta_{i}$ is 0 if joint $i$ does not intersect with a neighbor and 1 if it does intersect.

6) In order to find a minimum $\left(\omega^{3}\right)^{2}$ and zero $e$, we use the following design variables: The ellipticity ratio $b / a$, the order of the hyper-ellipse $k$, the inclination-angle at the apex $\theta$, and the additional twist-angles $\tau_{i}$. In practice the additional twist-angles are parameterized with the arc-length of the baseline and determined from a spline-function with 4 supports, thus adding 4 design variables to the optimization problem.

\section{COIL ENDS FOR THE SIS300}

The SIS300 dipoles will be wound from a cored Rutherfordtype cable which is more sensitive to hard-way bend than cable without stainless-steel core. Special care needs to be taken in the design of endspacers in order to minimize the integrated geodesic curvature in each block of cables. Further goals in the design of coil ends are

- the minimization of peak-field enhancement in the coils,

- the minimization of the physical magnet-length (maximization of the magnetic length of the magnet),

- and the minimization of unwanted multipoles from the coil ends.

The minimization of the geodesic curvature is achieved by the above-described method. The additional three objectives, which are related to the magnetic field in the magnet ends, are met in a subsequent optimization run, by the variation of the longitudinal position of the blocks in the coil ends.
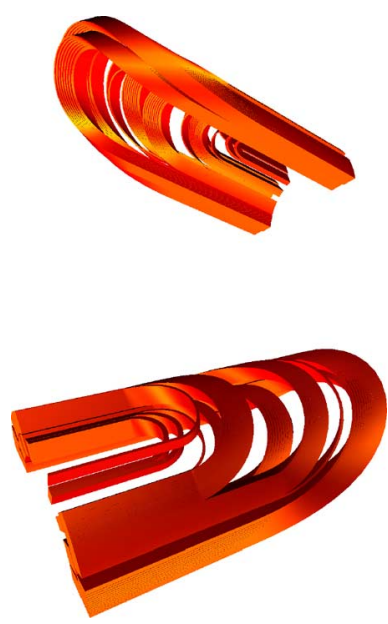

Fig. 9. Coil ends on the connection side SIS300 seen from different viewpoints.

The results of the optimization of the averaged field harmonics over the length of the coil end is given in units $10^{-4}$ at a reference radius of $40 \mathrm{~mm}$ :

\begin{tabular}{cccccc}
\hline \hline$n$ & units & $n$ & units & $n$ & units \\
\hline 3 & $<10^{-1}$ & 7 & $<10^{-1}$ & 11 & 6.7 \\
5 & $<10^{-1}$ & 9 & -0.8 & 13 & 0.7 \\
\hline \hline
\end{tabular}

Peak-field- and magnetic-length calculations will be available once the 3-D yoke design is finalized. The final coil-end design is shown in Fig. 9.

\section{CONCLUSION}

We have outlined a discrete theory of strips that serves as a model for superconducting cables in the coil ends of cosine-theta shaped coils. We have shown that the discrete model yields results that are equivalent to those of the previously validated continuous model. The advantage of discrete model lies in its low complexity as compared to the continuous model. The simplicity of the model results in an reduction of computing time, especially in optimization runs. The discrete design allows to model arbitrarily shaped busbar configuration in interconnection regions. The model was used to determine the mechanically optimal position of the cables in the coil ends of the SIS300 magnet.

\section{REFERENCES}

[1] R. Soika, M. D. Anerella, A. K. Gosh, P. Wanderer, M. N. Wilson, W. V. Hassenzahl, J. Kaugerts, and G. Moritz, "Inter-strand resistance measurements in cored Nb-Ti Rutherford cables.," IEEE Trans. Appl. Supercond., vol. 13, no. 2, pp. 2380-2383, Jun. 2003.

[2] J. M. Cook, "Strain energy minimization in SSC magnet winding," IEEE Trans. Magn., vol. 27, no. 2/4, pp. 1976-1980, Mar. 1991.

[3] B. Auchmann and S. Russenschuck, "Coil end design for superconducting magnets applying differential geometry methods," IEEE Trans. Magn., vol. 40, no. 2, pp. 1208-1211, Mar. 2003.

[4] W. Blaschke, Einführung in die Differentialgeometrie. Berlin: Springer Verlag, 1960.

[5] S. Russenschuck, Electromagnetic Design and Mathematical Optimization Methods in Magnet Technology. [Online]. Available: http://cern.ch/russ, 3rd ed. Feb. 2006 